\title{
The balance between excitation and inhibition controls the temporal organization of neuronal avalanches
}

\author{
F. Lombardi ${ }^{1}$, H. J. Herrmann ${ }^{1,2}$, C. Perrone-Capano ${ }^{3}$, D. Plenz ${ }^{4}$ and L. de Arcangelis ${ }^{5}$ \\ 1 Institute Computational Physics for Engineering Materials, ETH, Zürich, CH \\ 2 Departamento de Física, Universidade Federal do Ceará, 60451-970 Fortaleza, Ceará, Brazil \\ ${ }^{3}$ Biological Sciences Dept., Univ. of Naples Federico II and IGB-CNR, Napoli, Italy \\ 4 Section on Critical Brain Dynamics, NIH, Bethesda, Maryland 20892, USA \\ 5 Dept. of Information Engineering, Second University of Naples, Aversa (CE), Italy
}

\begin{abstract}
Neuronal avalanches, measured in vitro and in vivo, exhibit a robust critical behaviour. Their temporal organization hides the presence of correlations. Here we present experimental measurements of the waiting time distribution between successive avalanches in the rat cortex in vitro. This exhibits a non-monotonic behaviour, not usually found in other natural processes. Numerical simulations provide evidence that this behaviour is a consequence of the alternation between states of high and low activity, named up and down states, leading to a balance between excitation and inhibition controlled by a single parameter. During these periods both the single neuron state and the network excitability level, keeping memory of past activity, are tuned by homeostatic mechanisms.

PACS numbers: 05.65.+b, 05.45.Tp, 89.75.-k, 87.19.L-
\end{abstract}

Spontaneous neuronal activity can exhibit slow oscillations between bursty periods, or up-states, followed by substantially quiet periods. Bursts can last from a few to several hundreds of milliseconds and, if analysed at a finer temporal scale, have often shown a complex structure in terms of neuronal avalanches. In vitro experiments record avalanche activity [1, 2] from mature organotypic cultures of rat somatosensory cortex where they spontaneously emerge in superficial layers. The size and duration of neuronal avalanches follow power law distributions with stable exponents, which is a typical feature of a system in a critical state, where large fluctuations are present and the response does not have a characteristic size. The same critical behaviour has been measured in vivo from rat cortical layers during early post-natal development 3], from the cortex of awake adult rhesus monkeys [4], using microelectrode array recordings, as well as for dissociated neurons from rat hippocampus [5, [6] or leech ganglia [5] $]$. In vitro, quiet periods measured between bursts, also called down-states, can last up to several seconds. The emergence of these down-states can be attributed to various mechanisms: a decrease in the neurotransmitter released, either due to the exhaustion of available synaptic vesicles or to the increase of a factor inhibiting the release 7] such as the nucleoside adenosine [8], the blockade of receptor channels by the presence of external magnesium [9], or else spike adaptation [10]. A down-state is then characterized by a disfacilitation, i.e. absence of synaptic activity, of a large number of neurons causing long-lasting returns to resting potentials 11]. Recently, it was shown analytically and numerically that critical behaviour [12] characterizes up-states, whereas down-states are subcritical[13].

Whereas action potentials are rare during down-states, small amplitude depolarizing potentials, reminiscent of miniature potentials from spontaneous synaptic release, occur at higher frequencies. The non-linear amplification of small amplitude signals contributes to the generation of larger depolarizing events bringing the system back into the up-state, as observed in cortical slabs [14], dissociated cultures [15] and slice cultures [16]. The analysis of the amount of time striatal spiny neurons [17, 18] and cortical pyramidal neurons 19] spend at each value of the membrane potential shows that both cell types toggle between two preferred values [20]: a very negative one in the down state, and a more positive, depolarized one, in the up-state. The up-state being just a few millivolts from the action potential threshold, suggests that during the up-state neurons respond faster and more selectively to synaptic inputs. For cortical neurons the up-state would be a metastable state, i.e. the membrane potential would soon decay down to the resting potential value, if network mechanisms would not sustain the activity. The up-state has therefore network, rather than cellular, properties.

Here we focus on the temporal organization of neuronal avalanches both in organotypic cultures and neuronal networks simulations. Each avalanche $i$ is characterized by its starting and ending times, $t_{i}^{i}$ and $t_{i}^{f}$. The temporal organization is analysed by evaluating the distribution of waiting times $\Delta t_{i}=t_{i+1}^{i}-t_{i}^{f}$. This is a fundamental property of stochastic processes, widely investigated for natural phenomena and able to discriminate between a simple Poisson and a correlated process. Indeed, in the first case the distribution is exponential, whereas it exhibits a more complex behaviour with a power law regime if correlations are present. For a wide variety of phenomena, e.g. earthquakes and solar flares [21], human dynamics [22], biological systems [23], etc., this distribution always shows a monotonic behaviour. Recent results on freely behaving rats provide a lognormal size distribution and a monotonic waiting time distribution uniquely controlled by the average occurrence rate 24]. Anaesthetized rats, conversely, exhibit a more heavy-tailed size 


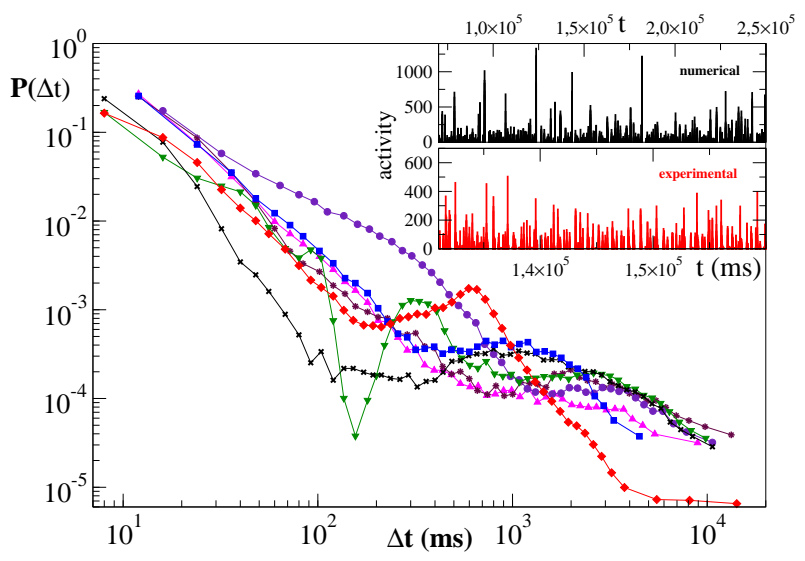

FIG. 1: (Color online) The distribution of waiting times for seven different slices of rat cortex. All curves show an initial power law regime between 10 and about $200 \mathrm{~ms}$, with an average exponent $2.15 \pm 0.32$. For $\Delta t>200 \mathrm{~ms}$ curves can become quite different with the common characteristics of a local minimum located at $200 \mathrm{~ms}<\Delta t_{\min }<1 \mathrm{~s}$, followed by a more or less pronounced maximum at $\Delta t \simeq 1-2 s$. In the insets two temporal sequences of neuronal activity for numerical (sum of potential variations) and experimental $(\mu V)$ data.

distribution and no universal scaling for the waiting time distribution. Here we show that the waiting time distribution for neuronal avalanches in vitro has an unusual non monotonic behavior. Numerical simulations on neuronal networks suggest that this is controlled by the slow alternation of up and down states, which determines both the network and the single neuron behavior.

Experiments were performed on coronal slices from rat dorsolateral cortex (postnatal day 0-2; $350 \mu \mathrm{m}$ thick) attached to a poly-D-lysine coated 60-microelectrode array (MEA; Multichannelsystems, Germany) and grown at $35.5{ }^{\circ} \mathrm{C}$ in normal atmosphere and standard culture medium without antibiotics for 4-6 weeks before recording. Avalanche activity was measured from cortexstriatum-substantia nigra triple cultures or single cortex cultures as reported previously[1]. Spontaneous avalanche activity is recorded outside the incubator in standard artificial cerebrospinal fluid (ACSF; laminar flow of $1 \mathrm{ml} / \mathrm{min}$ ) under stationary conditions for up to $10 \mathrm{hrs}$. The spontaneous local field potential (LFP) is sampled continuously at $1 \mathrm{kHz}$ at each electrode and lowpass filtered at $50 \mathrm{~Hz}$. Negative deflections in the LFP (nLFP) are detected by crossing a noise threshold of -3 SD (SD stands for standard deviation $\sim 3-5 \mu V$ ) followed by negative peak detection within $20 \mathrm{~ms}$. nLFP times and nLFP amplitudes are measured. Neuronal avalanches are defined as spatio-temporal clusters of nLFPs on the MEA 25]. A neuronal avalanche consists of a consecutive series of time bins of width $\delta t$ that contain at least one nLFP on any of the electrodes. Each avalanche is preceded and ended by at least one time bin with no activity. The waiting time $\Delta t$ is simply given by the number of empty bins between two successive avalanches times $\delta t$. Without loss of generality, the present analysis is done with $\delta t$ estimated for each culture as the average inter nLFP interval on the array and ranged between 3-6 ms for all cultures.

In Fig. 1 we show the waiting time distribution for different cultures of rat cortex slices. The curves exhibit a complex non-monotonic behaviour with common features: an initial power law regime and a local minimum followed by a more or less pronounced maximum. The presence of a power law implies that avalanche occurrence is not a pure Poisson process, namely successive avalanches are temporally correlated [26]. Moreover, the non-monotonic behaviour is not usually observed in natural phenomena. In order to investigate the origin of this behaviour, we simulate avalanche activity by a neuronal network model [27 29], which is able to reproduce the scaling properties of neuronal avalanches. Here we question whether and how the complex temporal organization of avalanches can be caused by the slow alternation between up-states and down-states. The basic idea is that after a large avalanche the involved neurons become hyperpolarized and the system goes into a downstate. Conversely, after a small avalanche active neurons remain depolarized, the system stays in an up-state.

We consider $N$ neurons at random positions, characterized by their potential $v_{i}$. Neurons are connected by a classical scale-free network [30], where a neuron $i$ has an out-going connectivity degree $k_{\text {out }_{i}}$. Once the network of output connections is established, we identify the resulting degree of in-connections, $k_{i n}$ for each neuron. To each synaptic connection we assign an initial random strength $g_{i j}$, with $g_{i j} \neq g_{j i}$, and to each neuron an excitatory or inhibitory character, with $10 \%$ inhibitory synapses. Whenever at a given time the value of the potential at a site $i$ is above a certain threshold, $v_{i} \geq v_{\max }$, the neuron sends action potentials which arrive to each of the $k_{\text {out }}$ pre-synaptic buttons. As a consequence, the total charge entering the connected neurons is $q_{i} \propto v_{i} k_{\text {out }_{i}}$, as in a firing rate based charge distribution. Each neuron receives charge in proportion to the synaptic strength $g_{i j}$, $v_{j}(t+1)=v_{j}(t) \pm \frac{q_{i}(t)}{k_{i n_{j}}} \frac{g_{i j}(t)}{\sum_{k} g_{i k}(t)}$, where the sum is on all out-going connections of $i$. Here the membrane potential variation is obtained by dividing the received charge by the surface of the soma of the post-synaptic neuron, proportional to the number of in-going terminals $k_{i n_{j}}$. The plus or minus sign is for excitatory or inhibitory $g_{i j}$, respectively. After firing, a neuron is set in a refractory state lasting 1 time step (about $10 \mathrm{~ms}$ ), during which it is unable to receive or transmit any charge. At the end of an avalanche, we implement Hebbian plasticity rules: The strength of the used connections between active neurons is increased proportionally to the activity of the synapse [31], namely the membrane potential variation of the post-synaptic neuron, $g_{i j}(t+1)=$ 
$g_{i j}(t)+\left(v_{j}(t+1)-v_{j}(t)\right) / v_{\max }$. Conversely, the strength of all inactive synapses is reduced by the average strength increase per bond, $\Delta g=\sum_{i j, t} \delta g_{i j}(t) / N_{b}$, where $N_{b}$ is the number of bonds. The presence of both strengthening and weakening rules implements a homeostatic regulatory mechanism for synaptic strengths, which underlies the system's critical behaviour. An external stimulus triggers further activity in the system: at the end of each avalanche the potential of a random neuron is increased by a small amount until another neuron gets at threshold and starts an avalanche. We implement the plasticity rules during a series of stimuli in order to modify the synaptic strengths, initially random. Previous studies have verified that the critical behaviour of avalanche distributions does not depend on parameter values or network properties and that this model reproduces quantitatively the background spectrum of measured EEG signals [27 29]. The implementation of a scale free network of connections in the present study is motivated by numerical convenience in terms of cpu time.

In order to implement the alternation between up and down-states, at the end of each avalanche we measure its size in terms of the sum of depolarizations $\delta v_{i}$ of all active neurons, $s_{\Delta v}=\sum \delta v_{i}$. If the last avalanche is larger than a threshold, $s_{\Delta v}>s_{\Delta v}^{m i n}$, the system transitions into a down-state and neurons active in the last avalanche become hyperpolarized proportionally to their previous activity, namely we reset

$$
v_{i}=v_{i}-h \delta v_{i}
$$

where $h>0$. This rule introduces a short range memory at the level of a single neuron and models the local inhibition experienced by a neuron, due to spike adaptation, adenosine accumulation, synaptic vesicle depletion, etc.

Conversely, if the avalanche just ended has a size $s_{\Delta v} \leq$ $s_{\Delta v}^{m i n}$, the system either will remain, or will transition into an up-state. All neurons firing in the previous avalanche are set to the depolarized value

$$
v_{i}=v_{\max }\left(1-s_{\Delta v} / s_{\Delta v}^{\min }\right)
$$

The neuron potential depends on the response of the whole network via $s_{\Delta v}$, in agreement with measurements of the neuronal membrane potential which remains close to the firing threshold in the up-state. $s_{\Delta v}^{\min }$ controls the extension of the up-state and therefore the level of excitability of the system. The high activity in the upstate must be sustained by collective effects in the network, otherwise the depolarized potentials would soon decay to zero, and therefore the random stimulation in the up-state has an amplitude that depends on past activity. Eqs. (1) and (2) each depend on a single parameter, $h$ and $s_{\Delta v}^{\min }$, which introduce a memory effect at the level of single neuron activity and the entire system, respectively. In order to reproduce the behavior observed experimentally, the parameters $s_{\Delta v}^{\min }$ and $h$ are

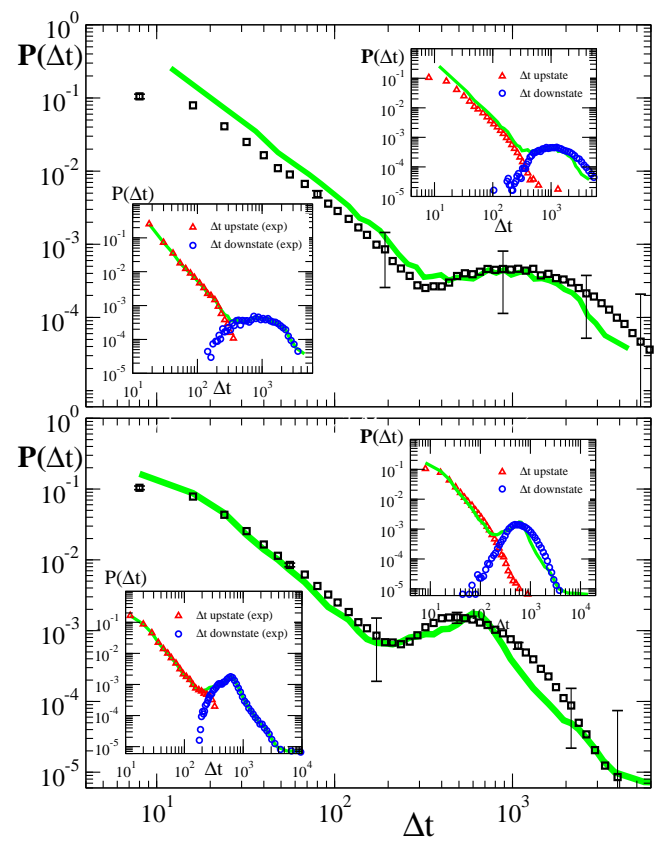

FIG. 2: (Color online) Waiting time distributions measured experimentally are compared with the average numerical distributions for 100 networks with $N=64000$ neurons. Top: numerical curve $\left(s_{\Delta v}^{\min }=140\right.$ and $\left.h=0.017\right)$ fitting the experimental curve with blue squares in Fig.1; Bottom: numerical curve $\left(s_{\Delta v}^{\min }=110\right.$ and $\left.h=0.02\right)$ fitting the experimental curve with red diamonds in Fig.1. In the insets the waiting time distribution evaluated separately in the up and downstate for the numerical (upper insets) and the experimental curves (lower insets). For the numerical curves, statistical error bars not shown are comparable to the symbol size.

controlled separately. Our simulations will show that the ratio $R=h / s_{\Delta v}^{\min }$ is the only relevant quantity controlling the temporal organization of avalanches.

Numerical simulations show that the system indeed switches between up and down states, with different temporal durations (insets of Fig.1). The numerical waiting time distributions (Fig.2) exhibit the non-monotonic behaviour of the experimental curves, where the position of the minimum is controlled by the value of $s_{\Delta v}^{\min }$ and the power law regime scales with the same exponent $\sim-2$ as experimental data. The agreement between the numerical and the experimental distribution is confirmed by the Kolmogorov-Smirnov test at a $p=0.05$ significance level. Both distributions pass the statistical test with $p=0.99$ (bottom panel) and $p=0.68$ (top panel). The different contribution from the two states is reflected in the activity temporal scale (insets of Fig.2). The upstate generates strongly clustered avalanches, originating the power law regime of the waiting time distribution, whose extension depends on $s_{\Delta v}^{m i n}$. Large $\Delta t$ between avalanches generated in the upstate are observed with a very small probability, which increases with decreasing $h$. Conversely, the waiting time distribution evaluated 
in the down-state has a bell-shaped behaviour centered at large intertimes which depends on $h$, i.e. for a larger disfacilitation of the network the probability to observe intermediate waiting times decreases in favour of long $\Delta t$.

The presence of the minimum and the height of the relative maximum are sample dependent (Fig.1) and for each sample the agreement between numerical and experimental data depends on the subtle balance between excitation and inhibition. For different samples, optimal agreement is realized when the ratio $R=h / s_{\Delta v}^{\min } \simeq 10^{-4}$. For instance, enhancing excitation, by increasing the threshold value $s_{\Delta v}^{\min }$, clearly produces a major shift in the data (Fig.3). Increasing inhibition, by increasing the parameter $h$, generates the opposite effect, recovering the good agreement with experimental data. Interestingly, the avalanche size and duration distributions also reproduce the experimental scaling behaviour for the parameter values expressing the balance between excitatory and inhibitory components. The abrupt transition between the up and down-state, controlled by a threshold mechanism, generates the minimum observed experimentally. Simulations of up-states and down-states only in terms of different external drives, without the single neuron state dependent behaviour (Eq.s 1-2), provide a monotonic waiting time distribution (inset Fig.3).

This complex non-monotonic behaviour, controlled by the system balance level between excitation and inhibition expressed by the parameter $R$, does not simply depend on the occurrence rate. The different behaviour with respect to alive rats 24] could be attributed to a larger separation in characteristic temporal scales between up and down-states. Indeed, long lasting downstates in our case originate waiting times one order of magnitude longer than for awake rats. Avalanches are temporally correlated in the up-state, whereas downstates are long term recovery periods where memory of past activity is erased. A detailed analysis of power spectra may shed further light on the temporal features of this alternation. The good agreement with experimental data indicates that the transition from an up-state to a down-state has a high degree of synchronization. Moreover it confirms that alternation between up and down-states is the expression of a homeostatic regulation which, during periods of high activity, is activated to control the excitability of the system and avoid pathological behaviour. The model suggests that the crucial feature of this temporal evolution is the different single neuron behaviour in the two phases: These collective effects must be supported by the single neuron behaviour, which toggles between two preferential states, a depolarized one in the up-state and a hyperpolarized one in the down-state. The model suggests that the depolarized neuron state is a network effect: the avalanche activity itself determines how close to the firing threshold a neuron stays in the up-state. Conversely, the hyperpolarized state is a form of temporal auto-correlation in the neuron activity. The

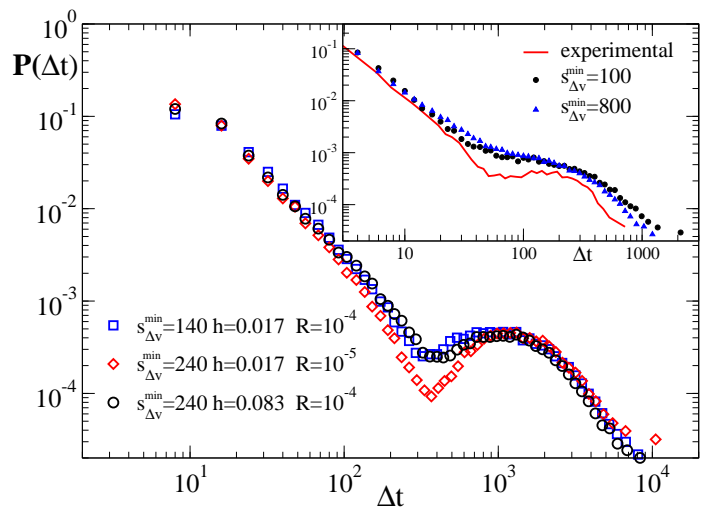

FIG. 3: (Color online) Waiting time distribution measured numerically for 100 networks of $N=16000$ neurons with different $s_{\Delta v}^{\min }$ and $h$. The best agreement is obtained for $R \simeq 10^{-4}$. In the inset waiting time distributions obtained with different stimulations in the up and down-states and without the single neuron state behaviour $(h=0)$.

critical state realizes the correct balance between excitation and inhibition via these self-regulating mechanisms.

Acknowledgments. We thank the SNF for funding within project 205321-13874. DP is supported by the Intramural Research Program of the NIMH, NIH.

[1] J.M. Beggs, D. Plenz, J. Neurosci. 23, 11167 (2003).

[2] J.M. Beggs, D. Plenz, J. Neurosci. 24, 5216 (2004).

[3] E.D. Gireesh, D. Plenz, Proc. Nat. Acad. Sci. USA 105, 7576 (2008).

[4] T. Petermann et al, Proc. Nat. Acad. Sci. USA 106, 15921 (2009).

[5] A. Mazzoni et al, PLoS ONE 2:e439 (2007).

[6] V. Pasquale et al, Neuroscience 153, 1354 (2008).

[7] K.J. Staley et al, Nature Neurosci. 1, 201 (1998).

[8] S.M. Thompson et al, J. of Physiol. 451, 347 (1992).

[9] E. Maeda et al, J. Neurosci. 15, 6835 (1995).

[10] M.V. Sanchez-Vives et al, J. Neurosci. 20, 4286 (2000).

[11] I. Timofeev et al, Proc. Nat. Acad. Sci. USA 98, 1924 (2001).

[12] A. Levina et al, Nature Phys. 3, 857 (2007); Phys. Rev. Lett. 102, 118110, (2009).

[13] D. Millman et al, Nature Phys. 6, 801 (2010).

[14] I. Timofeev et al, Cer. Cortex 10, 1185 (2000).

[15] D. Eytan, S. Marom, J. Neurosci. 26, 8465 (2006).

[16] D. Plenz, A. Aertsen, Neuroscience 70, 893 (1996).

[17] D. Plenz, S.T. Kitai, J. Neurosci. 18, 266 (1998).

[18] E.A. Stern et al, Nature 394, 475 (1998).

[19] M.O. Cunningham et al, Proc. Nat. Acad. Sci. USA 103, 5597 (2006).

[20] A. Hasenstaub et al, J. Neurosci. 27, 9607 (2007).

[21] L. de Arcangelis et al, Phys. Rev. Lett. 96, 051102 (2006).

[22] A.L. Barabasi, Nature 435, 207 (2005); D. Rybski et al, Proc. Nat. Acad. Sci. U.S.A. 106, 12640 (2009).

[23] J. Brujic et al, Nature Physics 269, 282 (2006).

[24] T.L. Ribeiro et al, PLoS ONE 5:e14129 (2010).

[25] D. Plenz, D.R. Chialvo, arXiv:0912.5369 
[26] T. Utsu, International Handbook of Earthquake and Engineering Seismology, 81A, 719 (2002).

[27] L. de Arcangelis et al, Phys. Rev. Lett. 96, 028107 (2006).

[28] G.L. Pellegrini et al, Phys. Rev. E 76, 016107 (2007).

[29] L. de Arcangelis, H.J. Herrmann, Proc. Natl. Acad. Sci.
USA 107, 3977 (2010).

[30] V.M. Eguiluz et al, Phys. Rev. Lett. 94, 018102 (2005).

[31] S.J. Cooper, Neurosci. Biobehav. Rev. 28, 851 (2005). 